VOL. 56 (1997) [345-347]

\title{
Computational studies of optimal controls
}

\author{
Heung Wing Joseph LeE
}

In this thesis, new computational methods are developed for several classes of optimal control problems. These problems are first discussed in Chapter 1, including their practical motivations. Furthermore, brief reviews on existing results which are directly relevant to the subject matter of the thesis are given.

For some optimal control problems, it is a difficult task to find an initial guess to the control such that the system of the corresponding differential equations can be successfully solved numerically. Without such a workable initial guess, all optimal control algorithms are powerless. For this reason, it is important to have a systematic way to obtain a workable initial guess. In Chapter 2, we address this problem by introducing a simple intuitive "Blind Man" algorithm. For illustration, we consider an optimal control problem involving a system of multi-link vertical planar robot arms moving under the effect of gravity and applied torques. This system can exhibit chaotic behaviour. Thus, a non-workable initial guess of torques can cause integration failure regardless of what numerical packages are used. We apply the "Blind Man" algorithm to obtain a workable initial guess. Then, the control parametrization algorithm is used to obtain an optimal solution.

In Chapter 3, a novel problem transformation called the Control Parametrization Enhancing Transform (CPET) is introduced for time optimal control problems. The transformed problem is an optimal control problem involving a piecewise constant function with pre-fixed switching points. Thus, it is readily solvable by the control parametrization algorithm. Switching times and singular control values of the original problem can then be calculated easily from the optimal solution of the transformed problem. For illustration, non-trivial examples are solved using the proposed method.

In Chapter 4, we consider a class of optimal control problems involving discrete control values. These problems are referred to as optimal discrete-valued control problems. Since the range set of the control function is a discrete set and hence not convex, the optimal discrete-valued control problems are, in fact, nonlinear combinatorial optimization problems. Using the idea of the control parametrization enhancing technique

Received 7th April, 1997

Thesis submitted to The University of Western Australia, November 1996. Degree approved, March 1997. Supervisor: Professor K.L. Teo.

Copyright Clearance Centre, Inc. Serial-fee code: 0004-9729/97 $\$ A 2.00+0.00$. 
developed in Chapter 3, it is shown that the optimal discrete-valued control problems are equivalent to optimal control problems involving only the enhancing control function, which is piecewise constant with pre-fixed switching points. The transformed problems are readily solvable by the control parametrization algorithm. To avoid rapid variation of the control function, we also consider the case in which a cost is imposed on control variation. Finally, several examples are solved and the results obtained are compared with existing ones.

As a by-product of the CPET, we also develop a novel method for solving a general class of nonlinear mixed discrete programming problems. Basically, we use the idea of CPET to convert nonlinear mixed discrete programming problems into equivalent standard optimization problems involving only continuous values. Numerical experiments, performed to demonstrate the effectiveness of the method, show that this method is superior to the existing ones.

In Chapter 5, we consider a class of nonlinear optimal control problems involving nonlinear affine dynamical systems and quadratic cost functionals. The Maximum Principle is used to derive the form of an optimal feedback controller. To compute this optimal feedback control law, a two-point boundary-value problem consisiting of $n$ state equations and $n^{2}$ 'Riccati-like' equations needs to be solved. This is a very difficult task from a numerical viewpoint. Thus, a practical computational method is developed based on the concept of control parametrization together with cubic spline functions. Moreover, error bounds of the proposed method are provided. For illustration, a nontrivial example is solved using the proposed method.

We consider a class of fixed terminal time optimal control problems involving general nonlinear continuous dynamics in Chapter 6. To compute the optimal feedback control law, we evaluate several open-loop optimal control trajectories corresponding to different initial conditions. These optimal trajectories are obtained using the well established control parametrization technique. Adopting a previously proposed iterative multivariate interpolation method to scattered data, together with a newly developed cardinal product spline approach, we obtain the suboptimal feedback controller from the previously calculated optimal trajectory data. For illustration, several examples are solved using the proposed method.

In Chapter 7, we consider a class of constrained discrete-time optimal control problems involving general nonlinear dynamics with fixed terminal time. We extend the method of Chapter 6 to the problem under consideration. Taking advantage of the nature of discrete time systems, a substantial reduction in the computational effort of synthesising the feedback controller is made possible. For illustration, a numerical example is solved using the proposed method.

In Chapter 8 , we consider an optimal control problem involving targeting of chaotic 
systems in a global sense. We address this problem by introducing a mixed strategy global sub-optimal feedback control scheme. The idea is to partition the state space into 2 parts, namely, the target region and it complement. The proposed controller will take different forms depending on which partition of the state space the system is in. Simulation results are also provided to illustrate the proposed scheme.

In Chapter 9, we conclude the thesis by giving brief discussions on topics for future research.

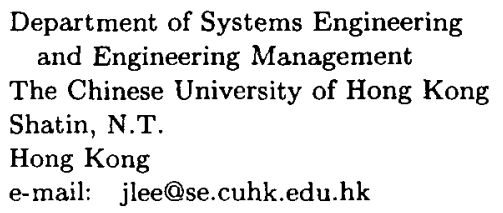

